\title{
The Behavior of Midsets When Repeatedly Taking the Midset of Two Lines in $\ell_{1}$ Geometry
}

\author{
Joshua M. Fitzhugh and David L. Farnsworth \\ School of Mathematical Sciences, Rochester Institute of Technology, Rochester, NY 14623, USA \\ Correspondence should be addressed to David L. Farnsworth; dlfsma@rit.edu
}

Received 22 September 2013; Accepted 15 April 2014; Published 8 May 2014

Academic Editor: Hernando Quevedo

Copyright (C) 2014 J. M. Fitzhugh and D. L. Farnsworth. This is an open access article distributed under the Creative Commons Attribution License, which permits unrestricted use, distribution, and reproduction in any medium, provided the original work is properly cited.

\begin{abstract}
We study the outcome of taking midsets of two lines in $\ell_{1}$ geometry. We establish the algorithm for repeatedly finding these midsets and characterize the limiting midsets. We discuss the issue of angle measurement in Minkowski geometries, especially with respect to the limiting midsets.
\end{abstract}

\section{Introduction}

An important class of planar Minkowski geometries are those that are determined by the unit circles $|x|^{p}+|y|^{p}=1$ for $p \geq 1$ $[1,2]$. These are designated $\ell_{p}^{2}$ or simply $\ell_{p}$ geometries. The most important example is Euclidean geometry with $p=2$. Our concern is with $\ell_{1}$ geometry, which is sometimes called taxicab, Manhattan, or city block geometry [3]. Our main interest is in the midsets of two lines in this geometry.

The $\ell_{1}$ midsets of two points or of a point and a line are less nuanced than the midsets of two lines. The $\ell_{1}$ midset of two points, which is all points equidistant from the two points, can have different shapes, but generally it is a piecewise linear set of three segments [3, pages 8-9]. The $\ell_{1}$ midset of a point and a line is an $\ell_{1}$ parabola, which has the appearance of an approximation of an Euclidean parabola by line segments [3, page 39$]$.

The midset of two lines is all points equidistant from the two lines. In Euclidean geometry, repeatedly taking midsets settles at the first or second step into a simple oscillation between pairs of orthogonal lines. The midset of the two intersecting and distinct lines $y=(\tan \varphi) x$ and $y=(\tan (\varphi+$ $\theta)) x$ is the perpendicular lines $y=(\tan (\varphi+\theta / 2)) x$ and $y=\left(\tan \left(90^{\circ}+\varphi+\theta / 2\right)\right) x$. Repeatedly finding midsets oscillates between these two orthogonal lines and a pair of lines rotated $45^{\circ}$ from them. If the original lines are orthogonal, that is, $\theta=90^{\circ}$, the oscillatory behavior begins at the first step. As seen in Section 2 , in $\ell_{1}$ geometry, repeatedly finding midsets of two lines generally does not produce this oscillatory behavior, unless the original lines are orthogonal. Subsequently, "midset" indicates " $\ell_{1}$ midset."

In Minkowski geometries, normality to a line is not reflexive; that is, the line perpendicular to the line that is perpendicular to the original line is not parallel to the original line, except in the case that the space's unit circle is a Radon curve [2, pages 233-234], [4, pages 137-138], and [5, page 145]. Analogously, we show that in general taking midsets is not reflexive in $\ell_{1}$ geometry; that is, the midset of the midset of two lines is not the original lines.

Without loss of generality, place the origin at the intersection of the original lines. In $\ell_{1}$ geometry the distance from a point to a line is measured either parallel to the $x$-axis or parallel to the $y$-axis. For $y=\alpha x$, if $|\alpha|>1$, then the distance is measured parallel to the $x$-axis; if $|\alpha|<1$, then the distance is measured parallel to the $y$-axis; and if $|\alpha|=1$, then either direction gives the same distance.

There are ten configurations for the original lines. They are labeled according to the location of their portion in the closed upper half plane. For our purposes, octants are open sets that do not include their boundaries, which are the axes and the lines $y= \pm x$. The configurations are as follows.

(1) The lines are in octants separated by another octant.

(2) The lines are $y=x$ and $y=-x$, or the lines are the axes. 
(3) One line is either $y=x$ or $y=-x$ and the other line is an axis.

(4) Both lines are in one octant.

(5) The lines are in neighboring octants in adjacent quadrants or separated by two octants.

(6) The lines are in neighboring octants in the same quadrant.

(7) One line is an axis and the other line is in a nonadjacent octant.

(8) One line is an axis and the other line is in an adjacent octant.

(9) One line is either $y=x$ or $y=-x$ and the other line is in a nonadjacent octant.

(10) One line is either $y=x$ or $y=-x$ and the other line is in an adjacent octant.

In Section 2, we show that Configuration 1 is a kind of attractor when repeatedly taking midsets of two lines. In Section 3, we establish the algorithm for finding midsets of lines in Configuration 1 and characterize the limiting midsets. Perhaps somewhat surprisingly, in most cases these limiting midsets are not the geometry's natural lines of symmetry, which are the axes and $y= \pm x$. In Section 4, we discuss the issue of angle measurement in Minkowski geometries, especially with respect to the limiting midsets.

\section{Finding Midsets of Two Lines}

We show that Configuration 1 is a kind of attractor when repeatedly taking midsets of two lines in any configuration. Within no more than three steps, iteratively taking midsets of lines in any configuration leads to a set in Configuration 1 or 2. We show that the midset of lines in Configuration 1 is also in Configuration 1. Since Configuration 2 is simple, only the properties of lines initially in Configuration 1 are discussed in Sections 3 and 4.

Theorem 1. For lines in Configuration 1, the midset is in Configuration 1, and the midset of the midset is in Configuration 1 in the original octants.

Proof. Without loss of generality take the lines

$$
y=\alpha x, \quad y=\beta x
$$

with $0<\alpha<1$ and $\beta<-1$. The midset of (1) is the lines

$$
y=\delta x=\frac{\beta(\alpha-1)}{\beta-1} x, \quad y=\varepsilon x=\frac{\beta(\alpha+1)}{\beta+1} x
$$

with $-1<\delta<0$ and $\varepsilon>1$. The midset is in Configuration 1 , but in octants different from the original lines. The midset of (2) is

$$
\begin{aligned}
& y=\eta x=\frac{\varepsilon(\delta+1)}{\varepsilon+1} x=\frac{\beta(\alpha+1)(\alpha \beta-1)}{(\beta-1)(\alpha \beta+1+2 \beta)} x, \\
& y=\kappa x=\frac{\varepsilon(\delta-1)}{\varepsilon-1} x=\frac{\beta(\alpha+1)(\alpha \beta+1-2 \beta)}{(\beta-1)(\alpha \beta-1)} x
\end{aligned}
$$

with $0<\eta<1$ and $\kappa<-1$, which is in Configuration 1 in the same octants as the original lines.

Theorem 2. For initial lines in Configuration 1, the midset of the midset is the original lines if and only if the original lines are orthogonal in the Euclidean sense. If the original lines are orthogonal in the Euclidean sense, then their midset is orthogonal lines that are rotated $45^{\circ}$ from the original pair of lines.

Proof. Setting $\eta=\alpha$ and $\kappa=\beta$ in (3) and (4), each gives the identity $\alpha^{2} \beta-\alpha \beta^{2}=\beta-\alpha$, and this is equivalent to $\alpha \beta=-1$. Hence, setting $\eta=\alpha$ gives $\kappa=\beta$, and vice versa. Substituting $-1 / \alpha$ for $\beta$ in (3) gives $\eta=\alpha$ and $\kappa=\beta=-1 / \alpha$. For the second statement in the theorem, set $\beta=-1 / \alpha$ in (2) to obtain $\delta=(\alpha-1) /(\alpha+1)$ and $\varepsilon=-(\alpha+1) /(\alpha-1)$ giving $\delta \varepsilon=-1$ and, following some trigonometry, the angle is $45^{\circ}$.

The following theorem can be seen with simple diagrams.

Theorem 3. For initial lines in Configuration 2, the midset of the lines $y=x$ and $y=-x$ is the axes, and the midset of the axes is the lines $y=x$ and $y=-x$.

Theorem 4. For initial lines in Configuration 3, the midset of the midset is in Configuration 1.

Proof. For a typical example, take the line $y=-x$ and the $x$ axis, whose midset is $y=-x / 2$ and the $y$-axis. The midset of those lines is $y=x / 2$ and $y=-3 x / 2$, which is in Configuration 1.

Theorem 5. Repeatedly taking midsets no more than three times of lines in Configurations 4 through 10 yields lines in Configurations 1 or 2.

Proof. For a typical example in Configuration 4, take $y=\alpha x$ and $y=\beta x$ for $0<\alpha<\beta<1$. The midset is $y=\delta x=[(\alpha+$ $\beta) / 2] x$ and the $y$-axis. The midset of this midset is $y=(\delta-1) x$ and $y=(\delta+1) x$, where $-1<\delta-1<0$ and $1<\delta+1<2$, which is in Configuration 1 .

For a typical example in Configuration 5, take $y=\alpha x$ and $y=\beta x$ for $0<\alpha<1$ and $-1<\beta<0$. The midset is $y=[(\alpha+\beta) / 2] x$ and the $y$-axis. As in Configuration 4 , the next midset is in Configuration 1.

For a typical example in Configuration 6, take $y=\alpha x$ and $y=\beta x$ for $0<\alpha<1$ and $\beta>1$, whose midset is $y=\delta x=$ $[\beta(\alpha+1) /(\beta+1)] x$ and $y=\varepsilon x=[\beta(\alpha-1) /(\beta-1)] x$, where $1 / 2<\delta<2$ and $\varepsilon<0$. This midset is in Configurations 1 or 5 , except for the special case $\alpha \beta=1$ for which $\delta=1$ and $\varepsilon=-1$, which is in Configuration 2.

Configurations 7-10 can be shown to produce Configuration 1 in no more than three steps by noting that typical examples for those configurations tighten an inequality used for Configurations 1 or 6 and subsequently yield lines in Configuration 1.

Since all configurations of initial lines lead to midsets in Configurations 1 or 2 within no more than three steps and 
since Configuration 2 is relatively simple, only Configuration 1 is discussed subsequently.

\section{Midsets for Configuration 1}

The algorithm for the sequence of slopes of the midsets of initial lines in Configuration 1 follows.

Algorithm 6. Beginning with the lines

$$
y=a_{0} x, \quad y=b_{0} x
$$

with $0<a_{0}<1$ and $b_{0}<-1$, the slopes of the first midset are

$$
a_{1}=\frac{b_{0}\left(a_{0}-1\right)}{b_{0}-1}, \quad b_{1}=\frac{b_{0}\left(a_{0}+1\right)}{b_{0}+1}
$$

with $-1<a_{1}<0$ and $b_{1}>1$. The slopes of the second midset, which is the midset of the first midset (6), are

$$
a_{2}=\frac{b_{1}\left(a_{1}+1\right)}{b_{1}+1}, \quad b_{2}=\frac{b_{1}\left(a_{1}-1\right)}{b_{1}-1}
$$

with $0<a_{2}<1$ and $b_{2}<-1$. The slopes of the third midset, which is the midset of the second midset, are

$$
a_{3}=\frac{b_{2}\left(a_{2}-1\right)}{b_{2}-1}, \quad b_{3}=\frac{b_{2}\left(a_{2}+1\right)}{b_{2}+1}
$$

with $-1<a_{3}<0$ and $b_{3}>1$. In general, for odd $n \geq 1$,

$$
a_{n}=\frac{b_{n-1}\left(a_{n-1}-1\right)}{b_{n-1}-1}, \quad b_{n}=\frac{b_{n-1}\left(a_{n-1}+1\right)}{b_{n-1}+1}
$$

with $-1<a_{n}<0$ and $b_{n}>1$. For even $n \geq 2$,

$$
a_{n}=\frac{b_{n-1}\left(a_{n-1}+1\right)}{b_{n-1}+1}, \quad b_{n}=\frac{b_{n-1}\left(a_{n-1}-1\right)}{b_{n-1}-1}
$$

with $0<a_{n}<1$ and $b_{n}<-1$.

Table 1 contains the positive angle of the limiting evennumbered midsets for initial lines at five evenly spaced angles $\theta_{0}$ from $4.5^{\circ}$ to $40.5^{\circ}$ incremented by $9.0^{\circ}$, giving slopes $a_{0}=$ $\tan \theta_{0}$, and at five evenly spaced angles $\varphi_{0}$ from $94.5^{\circ}$ to $130.5^{\circ}$ also incremented by $9.0^{\circ}$, giving slopes $b_{0}=\tan \varphi_{0}$. The angle of the second line in the limiting midset is obtained by adding $90^{\circ}$. The angles and slopes of the limiting odd-numbered midsets can be obtained by a $45^{\circ}$ rotation of the limiting lines of the even-numbered midsets. Limiting angles are rounded to two decimal places. No more than five pairs of taking midsets are required to obtain each tabulated value. For the entries on the main diagonal, the initial lines are orthogonal; that is, $\varphi_{0}-\theta_{0}=90^{\circ}$. Euclidean angle measure is used only for reference. Indeed, most formulas involve only the slopes of the lines. See Section 4 about angle measurement.

Theorem 7. If Algorithm 6 converges, it approaches a cycle of period two, alternating between pairs of orthogonal lines.
TABLE 1: Limiting angles in degrees are given for even-numbered midsets for selected lines $y=a_{0} x=\left(\tan \theta_{0}\right) x$ and $y=b_{0} x=$ $\left(\tan \varphi_{0}\right) x$ in Configuration 1.

\begin{tabular}{lccccc}
\hline$\theta_{0} \backslash \varphi_{0}$ & 94.5 & 103.5 & 112.5 & 121.5 & 130.5 \\
\hline 4.5 & 4.50 & 9.10 & 14.11 & 19.91 & 27.10 \\
13.5 & 9.10 & 13.50 & 18.20 & 23.53 & 30.01 \\
22.5 & 14.11 & 18.20 & 22.50 & 27.31 & 33.07 \\
31.5 & 19.91 & 23.53 & 27.31 & 31.50 & 36.46 \\
40.5 & 27.10 & 30.01 & 33.07 & 36.46 & 40.50 \\
\hline
\end{tabular}

Proof. Consider $n$ even. The proof for $n$ odd is almost identical. From (10)

$$
a_{n+2}=\frac{b_{n+1}\left(a_{n+1}+1\right)}{b_{n+1}+1}, \quad b_{n+2}=\frac{b_{n+1}\left(a_{n+1}-1\right)}{b_{n+1}-1}
$$

and from (9)

$$
a_{n+1}=\frac{b_{n}\left(a_{n}-1\right)}{b_{n}-1}, \quad b_{n+1}=\frac{b_{n}\left(a_{n}+1\right)}{b_{n}+1} .
$$

Substituting (12) into (11) gives

$$
\begin{gathered}
a_{n+2}=\frac{b_{n}\left(a_{n}+1\right)\left(a_{n} b_{n}-1\right)}{\left(b_{n}-1\right)\left(a_{n} b_{n}+1+2 b_{n}\right)}, \\
b_{n+2}=\frac{b_{n}\left(a_{n}+1\right)\left(a_{n} b_{n}+1-2 b_{n}\right)}{\left(b_{n}-1\right)\left(a_{n} b_{n}-1\right)} .
\end{gathered}
$$

In (13) set $a_{n+2}=a_{n}$, and in (14) set $b_{n+2}=b_{n}$. Each of these gives $b_{n}=-1 / a_{n}$.

The slopes of the limiting two lines are designated by $a$ and $b$. It may be somewhat surprising that in most cases the limiting midsets are not the geometry's natural lines of symmetry, which are the axes and $y= \pm x$.

An interesting symmetry in the outcomes of the iterative process is illustrated by the symmetry about the main diagonal in Table 1. If the initial lines are in Configuration 1, then $-1<a_{0} b_{0}<0, a_{0} b_{0}<-1$, or $a_{0} b_{0}=-1$. For the last condition, Theorem 2 says that the iterative process of finding midsets alternates between the initial lines and a pair of lines obtained by a $45^{\circ}$ rotation. In Table 1 , instances are on the main diagonal.

The condition $-1<a_{0} b_{0}<0$ corresponds to $90^{\circ}<\varphi_{0}-$ $\theta_{0}<135^{\circ}$, and examples are above the main diagonal in Table 1. For $a_{0} b_{0}<-1,45^{\circ}<\varphi_{0}-\theta_{0}<90^{\circ}$ and examples are below the main diagonal in Table 1 . To distinguish initial lines and outcomes of iterations for these two cases, we introduce primes for those with initial lines with $a_{0} b_{0}<-1$. That is, use $\theta_{0}^{\prime}, \varphi_{0}^{\prime}, a_{0}^{\prime}, b_{0}^{\prime}, a_{n}^{\prime}, b_{n}^{\prime}, a^{\prime}$, and $b^{\prime}$.

Theorem 8. Start with the two initial lines $y=a_{0} x$ and $y=$ $b_{0} x$ with $-1<a_{0} b_{0}<0$ corresponding to $90^{\circ}<\varphi_{0}-\theta_{0}<135^{\circ}$, and further two initial lines $y=a_{0}^{\prime} x$ and $y=b_{0}^{\prime} x$ with $a_{0}^{\prime} b_{0}^{\prime}<$ -1 corresponding to $45^{\circ}<\varphi_{0}^{\prime}-\theta_{0}^{\prime}<90^{\circ}$. Assume further the identities $\theta_{0}^{\prime}=\varphi_{0}-90^{\circ}$ and $\varphi_{0}^{\prime}=\theta_{0}+90^{\circ}$. Then $a_{n}^{\prime}=-1 / b_{n}$ and $b_{n}^{\prime}=-1 / a_{n}$ for all even $n \geq 0$. 
Proof. We use mathematical induction. For $n=0, a_{0}^{\prime}=$ $\tan \theta_{0}^{\prime}=\tan \left(\varphi_{0}-90^{\circ}\right)=-1 / \tan \varphi_{0}=-1 / b_{0}$. Similarly, $b_{0}^{\prime}=$ $-1 / a_{0}$. Assume that

$$
a_{n}^{\prime}=-\frac{1}{b_{n}}, \quad b_{n}^{\prime}=-\frac{1}{a_{n}}
$$

for $n$ even. From (13) and (14)

$$
\begin{gathered}
a_{n+2}^{\prime}=\frac{b_{n}^{\prime}\left(a_{n}^{\prime}+1\right)\left(a_{n}^{\prime} b_{n}^{\prime}-1\right)}{\left(b_{n}^{\prime}-1\right)\left(a_{n}^{\prime} b_{n}^{\prime}+1+2 b_{n}^{\prime}\right)}, \\
b_{n+2}^{\prime}=\frac{b_{n}^{\prime}\left(a_{n}^{\prime}+1\right)\left(a_{n}^{\prime} b_{n}^{\prime}+1-2 b_{n}^{\prime}\right)}{\left(b_{n}^{\prime}-1\right)\left(a_{n}^{\prime} b_{n}^{\prime}-1\right)} .
\end{gathered}
$$

Substituting (15) into (16) and using (13) and (14) give $a_{n+2}^{\prime}=$ $-1 / b_{n+2}$ and $b_{n+2}^{\prime}=-1 / a_{n+2}$.

Theorem 9. If $-1<a_{0} b_{0}<0$, then for $n$ even, $a_{n+2}>a_{n}$, $b_{n+2}<b_{n}$, and $-1<a_{n+2} b_{n+2}<a_{n} b_{n}$, and the angle $\varphi_{n}-\theta_{n}$ between the midset's lines is monotonically decreasing toward $90^{\circ}$. If $a_{0}^{\prime} b_{0}^{\prime}<-1$, then for $n$ even, $a_{n+2}^{\prime}<a_{n}^{\prime}, b_{n+2}^{\prime}>b_{n}^{\prime}$, and $a_{n}^{\prime} b_{n}^{\prime}<a_{n+2}^{\prime} b_{n+2}^{\prime}<-1$, and the angle $\varphi_{n}^{\prime}-\theta_{n}^{\prime}$ between the midset's lines is monotonically increasing toward $90^{\circ}$. Symmetrically placed initial lines have the same pairs of orthogonal lines in the limit, which always exists.

Proof. The proof for the case $-1<a_{0} b_{0}<0$ is outlined. The other case's proof is very similar. Using (13) with $n=0$, set $a_{2}>a_{0}$, which reduces to $a_{0} b_{0}>-1$. Reversing the algebraic steps gives $a_{2}>a_{0}$. Similarly, using (14) with $n=0$, set $b_{2}<b_{0}$, which reduces to $a_{0} b_{0}>-1$. Reversing the steps gives $b_{2}<b_{0}$. To see that $-1<a_{2} b_{2}$, for $n=0$ multiply (13) and (14), which becomes

$$
a_{2} b_{2}=\frac{b_{0}^{2}\left(a_{0}+1\right)^{2}\left(a_{0} b_{0}+1-2 b_{0}\right)}{\left(b_{0}-1\right)^{2}\left(a_{0} b_{0}+1+2 b_{0}\right)}
$$

This is seen to be greater than -1 by setting it greater than -1 and performing the reversible steps of cross-multiplying by the negative denominator, expanding, cancelling terms, and factoring, giving

$$
\left(a_{0} b_{0}+1\right)\left(a_{0}^{2} b_{0}^{2}+1-2 b_{0}^{2}\right)<0 .
$$

The first factor is positive since $-1<a_{0} b_{0}$, and the second factor is negative for $-1<a_{0} b_{0}<0$ and $b_{0}<-1$. Mathematical induction on $n$ gives the results for even $n$. The slopes $\left\{a_{n}\right\}$ are an increasing sequence, which is bounded above, since $a_{n}<1$ for all $n$. The limiting slope exists, and, using Theorems 7 and 8 , the process of taking midsets monotonically approaches a cycle alternating between pairs of orthogonal lines, since $a^{\prime}=-1 / b=-1 /(-1 / a)=a$.

\section{Definitions of $\ell_{1}$ Angle and Angle Bisector}

Thompson discusses at length the "ambiguity about the definition of angle" [1, page 273], and Busemann declares the "great arbitrariness of angle measure" [6, page 275]. Angle measure remains an active area of study in geometry and analysis [7-10]. Trigonometric functions are generally defined in terms of lines, rays, or vectors. For example, calling them vectors, the cosine of a pair is the dot product of the vectors divided by the product of their lengths. Angle measure is virtually unique in Euclidean geometry, but there are many natural choices in Minkowski geometry, depending upon the properties that are desirable. For example, in $\ell_{1}$ geometry, the angle between $(x, y)$ on the unit circle near $(1,0)$ is $2 y$, if the angle is defined by the arc length from the $x$-axis along the unit circle. Defining the sine function as the length of the opposite side divided by the hypotenuse in $\ell_{1}$ measure, the ratio of sine to the angle is the constant $1 / 2$. In order to preserve certain properties of derivatives of trigonometric functions with respect to their arguments, Thompson selects a measure of angle that preserves the property that the limit as the angle approaches zero of the sine divided by the angle is 1 [1, pages. 264-266]. Generally, the angle measure between two vectors or rays that share the same Euclidean angle measurement is different, since the size of the angle depends upon the specific directions of the vectors or rays.

Except for special situations, such as the lines being the axes, the limiting line $y=a x$ in Section 3 is not an angle bisector. Various definitions of angle give different angle bisectors. Here, as elsewhere, Euclidean angle measure is used only for easy reference. The slope is the fundamental quantity.

We consider four options of $\ell_{1}$ angle bisector for lines in Configuration 1.

(1) Use Euclidian $\left(\ell_{2}\right)$ angle measure and bisectors.

(2) Use $\ell_{1}$ angles based on the subtended arc length of the unit circle $|x|+|y|=1$ and their bisectors.

(3) Use the first step in Algorithm 6; that is, in Configuration 1 , use $y=b_{1} x$ in (4).

(4) Use the lines $y=a x$ obtained in the limit in Section 3.

Using Euclidian measure follows Busemann [6, page 277] and [11]. Option 2 is the same as using area measure, since in $\ell_{1}$ geometry the subtended arc length has a constant of proportionality with the Euclidean area.

For an example, take the lines $y=\left(\tan 4.5^{\circ}\right) x=0.07870 x$ and $y=\left(\tan 130.5^{\circ}\right) x=-1.171 x$ in Configuration 1, and compare the bisectors obtained in the second octant.

For Option 1, the Euclidean angle bisector has the equation $y=\left(\tan \left[\left(4.5^{\circ}+130.5^{\circ}\right) / 2\right]\right) x=\left(\tan 67.5^{\circ}\right) x=2.414 x$.

For Option 2, the $\ell_{1}$ unit circle centered at the origin is the square with vertices at $(1,0),(0,1),(-1,0)$, and $(0,-1)$. Its circumference is 8 . The $\ell_{1}$ length of the subtended arc along the portion $y=1-x$ of the $\ell_{1}$ unit circle between $y=0.07870 x$ and the positive $y$-axis is $2-2(1-[1 /(1+$ $0.07870)])=1.854$. The length of the subtended arc between $y=-1.171 x$ and the positive $y$-axis is $2[1 /(1+1.171)]=$ 0.9212 . One-half the length of the subtended arc between $y=0.07870 x$ and $y=-1.171 x$ is $(1.854+0.9212) / 2=1.388$. Add this length to the arc length between the $x$-axis and $y=$ $0.07870 x$ to obtain $2(1-[1 /(1+0.07870)])+1.388=1.534$. 
The tangent of the angle that this bisector makes with the $x$ axis is $[1.534 / 2] /[1-(1.534 / 2)]=3.292$, which corresponds to the angle $\tan ^{-1} 3.292=73.10^{\circ}$.

For Option $3, b_{1}=[-1.171 /(-0.171)](1.079)=7.389$, which corresponds to the angle $\tan ^{-1} 7.389=82.29^{\circ}$.

For Option 4, using the value in Table 1, the limiting line is $y=\left[\tan \left(27.10^{\circ}+45^{\circ}\right)\right] x=\left(\tan 72.10^{\circ}\right) x=3.096 x$.

Depending upon which option is selected, the angle that the bisector makes with the $x$-axis is $67.5^{\circ}, 73.10^{\circ}, 82.29^{\circ}$, or $72.10^{\circ}$, in this example.

\section{Conflict of Interests}

The authors declare that they have no conflict of interests regarding the publication of this paper.

\section{References}

[1] A. C. Thompson, Minkowski Geometry, Cambridge University Press, Cambridge, UK, 1996.

[2] R. V. Benson, Euclidean Geometry and Convexity, McGraw-Hill, New York, NY, USA, 1966.

[3] E. F. Krause, Taxicab Geometry, Dover, New York, NY, USA, 1986.

[4] H. Busemann and P. J. Kelly, Projective Geometry and Projective Metrics, Academic Press, New York, NY, USA, 1953.

[5] J. C. Á. Paiva and A. Thompson, "On the perimeter and area of the unit disc," The American Mathematical Monthly, vol. 112, no. 2, pp. 141-154, 2005.

[6] H. Busemann, Geometry of Geodesics, Academic Press, New York, NY, USA, 1955.

[7] B. V. Dekster, "An angle in Minkowski space," Journal of Geometry, vol. 80, no. 1-2, pp. 31-47, 2004.

[8] B. V. Dekster, "A metric space of directions in Minkowski space," Journal of Geometry, vol. 80, no. 1-2, pp. 48-64, 2004.

[9] V. W. Thürey, "A generalization of Euclidean angle," Journal of Convex Analysis, vol. 20, no. 4, pp. 1025-1042, 2013.

[10] V. W. Thürey, "Angles and a classification of normed spaces," Annals of Functional Analysis, vol. 4, no. 1, pp. 114-137, 2013.

[11] H. Busemann, "The foundations of Minkowskian geometry," Commentarii Mathematici Helvetici, vol. 24, no. 1, pp. 156-187, 1950. 


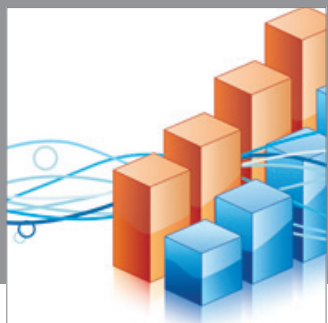

Advances in

Operations Research

mansans

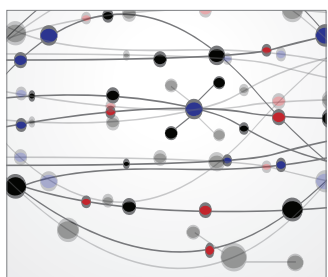

The Scientific World Journal
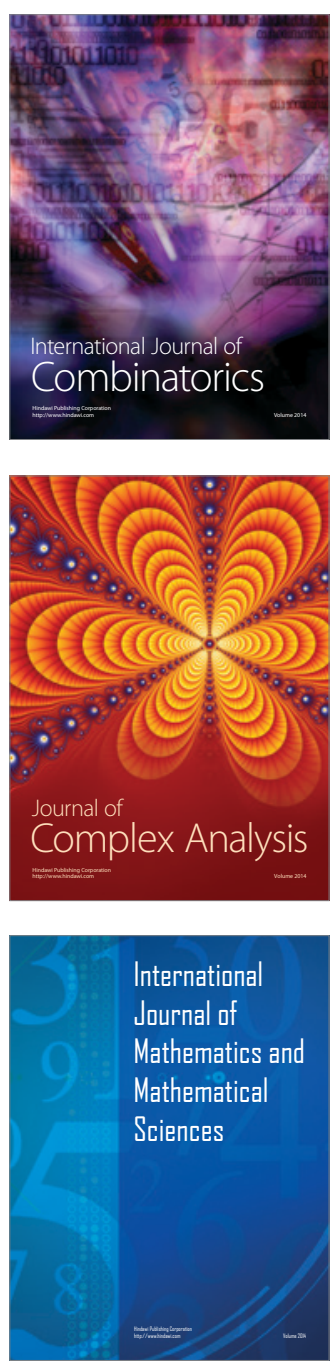
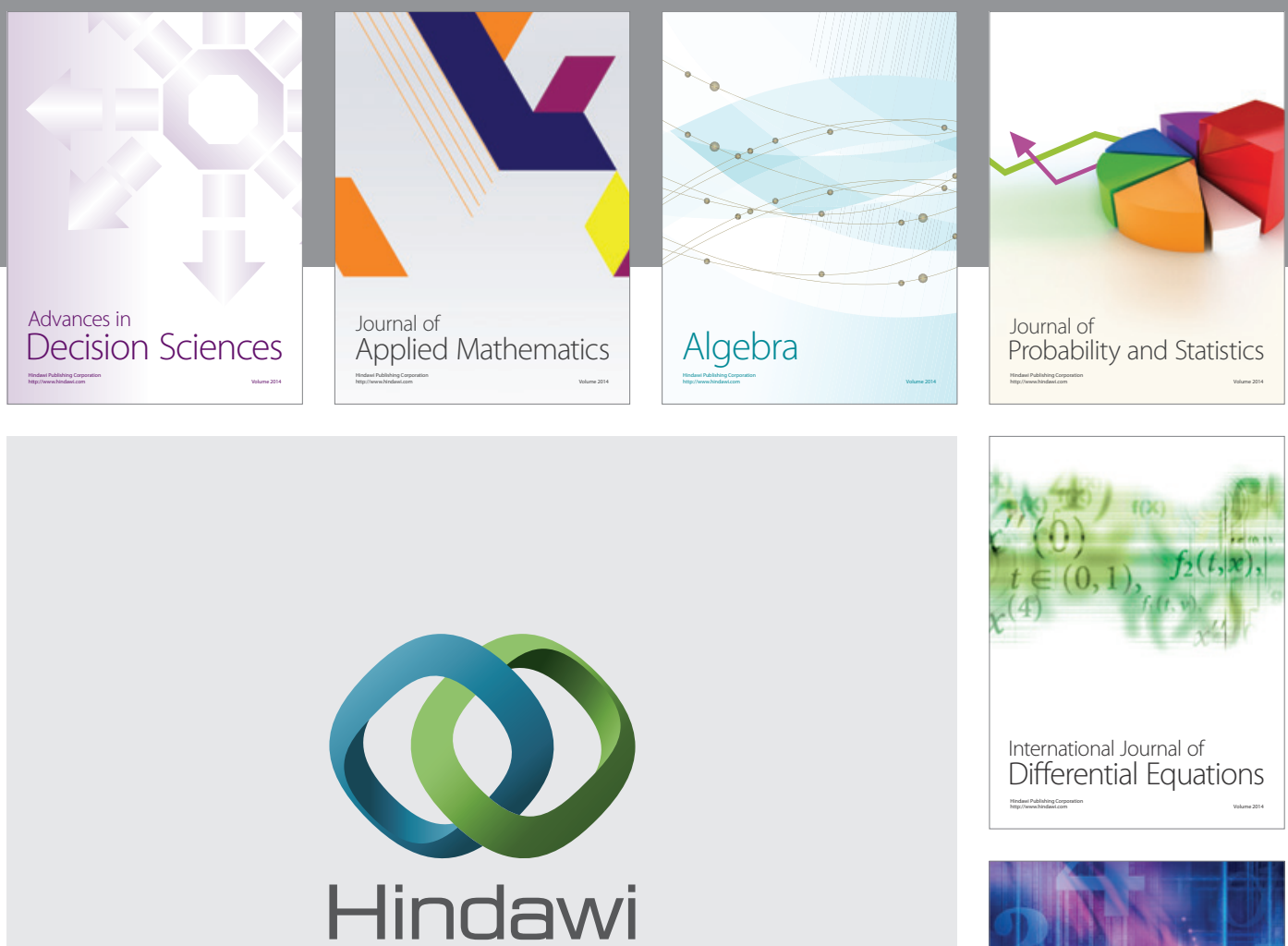

Submit your manuscripts at http://www.hindawi.com
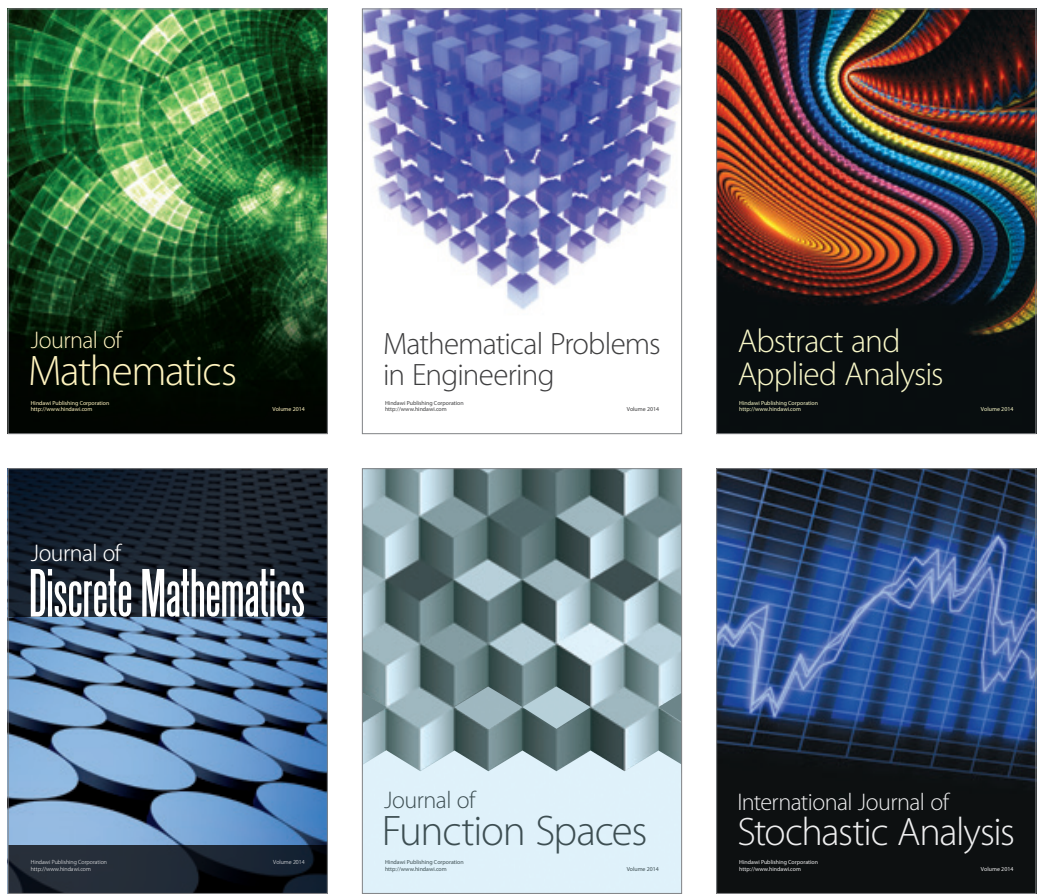

Journal of

Function Spaces

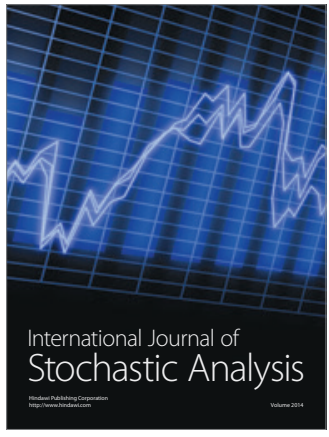

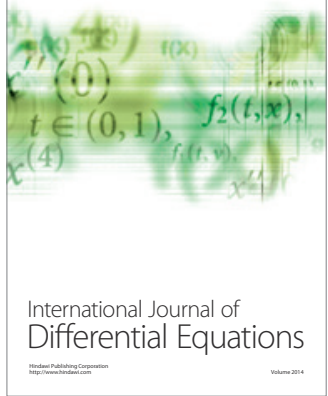
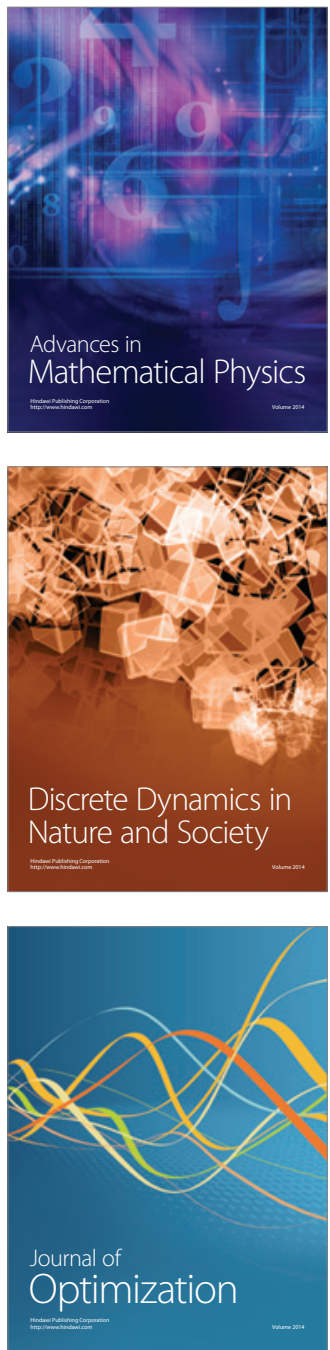